\title{
Multiple lymphomatous polyposis and the role of fine needle aspiration cytology
}

\author{
P.J. Carder and D.R. Gouldesbrough ${ }^{1}$
}

Department of Pathology, University of Edinburgh, Medical School, Teviot Place, Edinburgh EH8 9AG, and ${ }^{1}$ Department of Histopathology, Bradford Royal Infirmary, Duckworth Lane, Bradford BD9 6RJ, UK

\begin{abstract}
Summary: Multiple lymphomatous polyposis is an uncommon but distinctive form of gastrointestinal lymphoma. Clinical symptoms may closely resemble those of colorectal carcinoma and diagnostic confusion may result. The condition has a characteristic pathological appearance and immunophenotype which is important in allowing distinction from other less aggressive forms of gastrointestinal lymphoma. We report a case of this unusual condition in which the diagnosis was aided by fine needle aspiration cytology.
\end{abstract}

\section{Introduction}

Primary gastrointestinal lymphomas are uncommon, accounting for less than $4 \%$ of all gastrointestinal malignancies. ${ }^{1}$ The stomach is by far the commonest site involved although between 10 and $20 \%$ occur in the colorectum. ${ }^{2}$ Primary colorectal lymphoma is, however, distinctly unusual comprising only $0.2 \%$ of all colorectal malignancies in the United Kingdom. ${ }^{3}$ Unfortunately an increase in case number is to be anticipated following reports of an association with the acquired immunodeficiency syndrome, AIDS. ${ }^{4}$

Multiple lymphomatous polyposis (MLP) is a distinctive and particularly rare form of primary gastrointestinal lymphoma which tends to arise in the ileocaecum and has an especially aggressive clinical course. ${ }^{5}$ MLP has a characteristic nodular pattern of infiltration which may give rise to multiple colonic polyps but clinically the condition may be indistinguishable from colorectal carcinoma. In view of the need for chemotherapy, accurate diagnosis is essential. We present a case of this unusual tumour in which diagnosis was facilitated by fine needle aspiration cytology.

\section{Case report}

A 66 year old male presented with a 4 month history of colicky abdominal pain. He had no significant past medical history and was otherwise well. On examination an ill-defined, non-tender

Correspondence: D.R. Gouldesbrough, B.Sc., M.B., Ch.B., M.R.C.Path.

Accepted: 30 September 1992 right iliac fossa mass was palpated. The patient was undistressed.

Abdominal X-rays showed subacute small bowel obstruction and ultrasound revealed a caecal mass with enlarged mesenteric lymph nodes and left kidney. A barium enema confirmed a polypoid tumour in the caecum, probably a carcinoma. All other investigations were negative. A firm clinical diagnosis was made of caecal carcinoma with lymph node and renal metastases.

Fine needle aspiration of the enlarged left kidney was carried out and revealed a lymphoma but within 2 days, before this could be further characterized, the patient developed complete distal small bowel obstruction. At laparotomy there was complete obstruction of the ileocaecal valve by a polypoid tumour and a right hemicolectomy was performed. An additional segment of small intestine was also removed and the liver and left kidney biopsied.

\section{Pathology}

The fine needle aspirate contained a population of mildly pleomorphic enlarged lymphocytes which immunohistochemically stained with anti-CD22 and anti-IgM, and showed kappa light chain restriction. The findings indicated a $B$ cell lymphoma. Examination of the right hemicolectomy specimen revealed obstruction of the ileocaecal value by a substantial polypoid tumour arising within the valve and adjacent caecum and extending into distal terminal ileum. The mucosal surface of the small bowel was studded with numerous 
polyps which ranged in size up to $3 \mathrm{~cm}$ in maximum dimension.

Histology revealed a lymphoid infiltrate principally within mucosa and submucosa (Figure 1) with many of the cells having a centrocyte-like morphology. Local lymph nodes and hepatic portal triads were infiltrated by similar cells. In both small and large bowel a poorly defined nodular pattern was apparent with tumour cells surrounding residual germinal centres and partially replacing the pre-existing mantle zones. Lymphoepithelial lesions were not identified. Immunohistochemistry found the tumour cells to express CD45, CD22, surface IgM and IgD and kappa light chain. A small subset were positive for CD5.

\section{Discussion}

MLP is a rare form of primary gastrointestinal lymphoma characterized by polypoid accumulations of malignant lymphoid tissue principally within the submucosa. The characteristic morphological appearances were first described by Cornes in $1961 .^{6}$ Although initially thought that any type of lymphoma could give rise to this pattern of infiltration, it has recently become clear that MLP is a distinct form of centrocytic lymphoma ${ }^{7}$ with a tendency for systemic spread and an aggressive natural history.

MLP must be distinguished from colorectal carcinoma and other forms of gastrointestinal lymphoma. In our case a firm clinical diagnosis of caecal carcinoma was made following detection of a polypoid tumour on barium enema. This required reassessment in the light of an aspirate containing atypical monoclonal $B$ cells. Fine needle aspiration (FNA) is an accepted, relatively atraumatic technique for sampling intra-abdominal lesions. ${ }^{8}$ The scope of FNA in this context is enlarged by the role of immunocytochemistry in distinguishing neoplastic from reactive lymphoid populations. ${ }^{9}$ A final diagnosis of MLP, however, required detailed histological and immunocytochemical assessment of the resection specimen. The absence of lympho-

\section{References}

1. Loehr, W.J., Mujahed, Z., Zahn, F.D., Gray, G.F. \& Thornbjarnson, B. Primary lymphoma of the gastrointestinal tract: a review of 100 cases. Ann Surg 1969, 170: 232-238.

2. Contreary, K., Nance, F.C. \& Becker, W.F. Primary lymphoma of the gastrointestinal tract. Ann Surg 1980, 191: 593-598.

3. Shephard, N.A., Hall, P.A., Coates, P.J. \& Levison, D.A. Primary lymphoma of the colon and rectum. A histopathological and immunohistochemical analysis of 45 cases with clinicopathological correlations. Histopathology 1988, 12: 235-252.

4. Lee, M.H., Waxman, M. \& Gillooley, J.F. Primary malignant lymphoma of the anorectum in homosexual men. Dis Colon Rectum 1986, 29: 413-416.

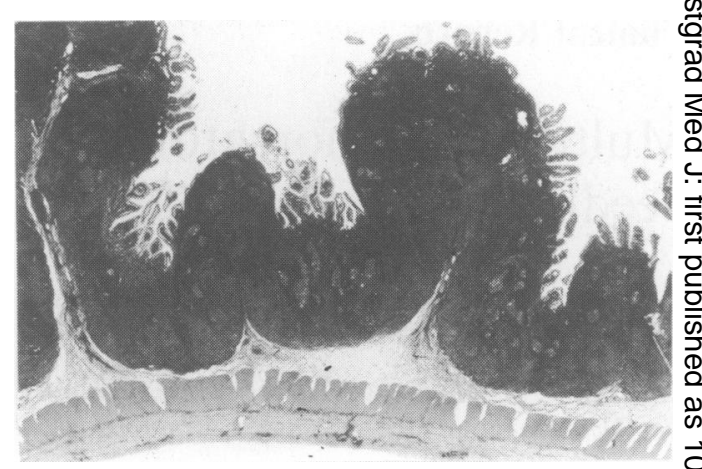

Figure 1 The lymphoid infiltrate of multiple lymphomatous polyposis is principally confined to the mucosa and submucosa.

epithelial lesions and the positive immunocytochemical staining with anti-CD5 are typical and allow distinction from other less aggressive forms of B cell lymphoma.

MLP is usually widespread throughout the gas- $\stackrel{2}{\rightarrow}$ trointestinal tract sometimes extending to involve $z$ the stomach ${ }^{5}$ and peripheral lymphadenopathy is common. Our case had evidence of systemic spread $\overrightarrow{0}$

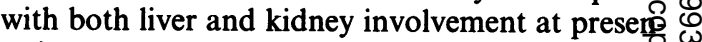
tation. In view of the likelihood of extensi disease, the treatment of choice for MLP is chem $\bar{P}$ therapy, for example, with chlorambucil, whilst other more localized lymphomas require radical surgery with or without radiotherapy. ${ }^{3}$ Accurate pathological diagnosis is therefore essential.

This case serves to re-emphasize the value of fine needle aspiration in the diagnosis of intra-abdominal lesions. It is also a useful reminder of how common symptomatology may occasionally mask unusual and unexpected pathology.

\section{Acknowledgement}

We wish to thank Mrs Diane White for her speed and accuracy in typing this manuscript.

5. Isaacson, P.G., Maclennan, K.A. \& Subbuswamy, S.G. Multi- N ple lymphomatous polyposis of the gastrointestinal tract. Histopathology 1984, 8: 641-656.

6. Cornes, J.S. Multiple lymphomatous polyposis of the gastrointestinal tract. Cancer 1961, 14: 249-257.

7. Blackshaw, A.J. Non Hodgkins lymphomas of the gut. In: Wright, R. (ed.) Recent Advances in Gastrointestinal Pathology. W.B. Saunders, Eastbourne, 1980, pp. 213-240.

8. Koss, L.G., Woyke, S. \& Olszewski, W. (eds) Aspiration Biopsy. Cytologic Interpretation and Histologic Basis. Igaku Shoin, New York, Tokyo, 1984, pp. 30-32.

9. Spriggs, A.I. \& Vanhegan, R.I. Cytologic diagnosis of lymphoma in serous effusions. J Clin Pathol 1981, 34: 1311-1325. 